\title{
MINIMAL INDIVIDUAL HAEMOSTASIS (MIH) - FEASIBILITY IN CONVENTIONAL AND DISTAL TRANS-RADIAL APPROACH
}

\author{
MAGED MOKBEL ${ }^{1}$, NICOLAE FLORESCU ${ }^{2}$, CRINA JULIETA SINESCU ${ }^{3}$ \\ 1,3 “Carol Davila” University of Medicine and Pharmacy, București, ${ }^{2}$ Polisano Clinic, Sibiu, ' "Sfântul Ioan”" Clinical Emergency Hospital, București, \\ 3 "Bagdasar Arseni”" Clinical Emergency Hospital, București
}

\author{
Keywords: PCI, \\ Haemostasis, Radial \\ approach, Distal \\ transradial, Snuff-box
}

\begin{abstract}
There is a growing use of trans-radial catheterisation for angiography and percutaneous interventions. The advantages of trans-radial over traditional femoral access include a decrease in the incidence of access site bleeding complications and improved patient comfort and recovery. Ensuring short haemostasis, which controls arterial bleeding after transracial interventions, but at the same time maintains radial arterial flow, appears to be the most important factor in reducing periprocedural complications, especially radial artery occlusion (RAO). We evaluated the technical feasibility of our gradual deflation haemostasis method in a population of patients treated by both conventional and distal trans-radial approach, within 1 month period. A feasibility assessment was performed for 101 patients to assess whether our hemostasis technique could be performed safely. We concluded that our technique is feasible after trans-radial approach, in both categories of access, conventional or distal. The rate of peri-procedural complications was low and related to the type of approach used, with more positive results on the distal trans-radial approach.
\end{abstract}

\section{INTRODUCTION}

There is a growing use of trans-radial catheterisation for angiography and percutaneous interventions. The advantages of trans-radial over traditional femoral access include a decrease in the incidence of access site bleeding complications and improved patient comfort and recovery. In recent years, there have been changes to conventional techniques of classical transradial approach, such as the distal trans-radial approach with a significant increase in the rate it's being used compared to femoral.(1)

Although rare, and predominantly asymptomatic, radial artery occlusion (RAO) denies the future use of radial artery for coronary interventions or as an arterial graft for coronary bypass surgery or for haemodialysis fistula.(2) RAO is not always benign, and ischaemia of the hand has been reported in the medical literature in a relatively small number of cases. Ensuring short haemostasis, which controls arterial bleeding but at the same time maintains radial arterial flow, appears to be the most important factor in reducing peri-procedural RAO.

Therefore, we wanted to evaluate the technical feasibility of our gradual deflation haemostasis method in a population of patients treated by conventional or distal transradial approach, within 1 month period.

MATERIALS AND METHODS
All adult patients underwent trans-radial
catheterisation at Polisano Clinic, Sibiu, Romania, between
January 2018 and February 2018 were considered included in
this study. The study data were analysed retrospectively and
formed part of data collected in controlled study related to the
distal trans-radial approach compared to the conventional trans-
radial approach and as an institutional initiative to improve
quality to reduce RAO at our center by implementing document

recommendations consensus on the standard of care.

A feasibility assessment was performed for 101 patients to assess whether HMI could be performed safely and consistently achieve more optimised haemostasis.

As part of a standard pre-procedural protocol of the institute, the basic permeability of the ulno-palmar arch was ensured using a combination of pulse oximetry and plethysmography, as described by Barbeau. Patients who did not show recovery from pulse follow-up within two minutes (type D response) were excluded from transradial access.

50 patients had the conventional transradial approach, but the other 51 patients had the distal transradial approach in the anatomical tabachera. Initial radial access, catheterization, and radial sheath insertion were similar in all patients following a catheterization laboratory protocol.

Patients were prepared and draped in a sterile manner and $2 \%$ lidocaine was administered subcutaneously over the planned site of puncture of the right radial artery, either the classical approach by the modified Seldinger technique. Either distal approach in the anatomical snuff using the technique without ultrasonographic guidance.

A 6 Fr radial sheath was then advanced into the radial artery lumen using a modified Seldinger technique.

A combination of $200 \mathrm{mcg}$ nitroglycerin, $2.5 \mathrm{mg}$ verapamil and 50 units $/ \mathrm{kg}$ unfractioned heparin (maximum 5,000 units) was administered in the radial artery, unless hypotension is prohibited. Coronary angiography was performed using 6 Fr diagnostic catheters and interventions using $6 \mathrm{Fr}$ guide catheters. Unfractioned heparin based on single weight was used followed by dose supplementation if percutaneous coronary intervention was performed according to European treatment guidelines.

The activated clotting time was checked to ensure a

${ }^{1}$ Corresponding author: Crina Juieta Sinescu, Şos. Berceni, Nr. 12, București, România, E-mail: crinasinescu@ gmail.com, Phone: +40722 300301. Article received on 20.09.2020 and accepted for publication on 03.04.2021 


\section{CLINICAL ASPECTS}

time of over 250 seconds in the patients undergoing the intervention. In all patients undergoing stent placement, aspirin $(325 \mathrm{mg})$ together with clopidogrel $(300 \mathrm{mg})$ or ticagrelor $(180$ $\mathrm{mg}$ ) were administered at the time of catheterisation, unless previously administered.

Immediately after the procedure, the sheath was withdrawn 2-3 cm and a TR Band TM (Terumo Medical) radial compression device was applied. TR-Band inflation with $20 \mathrm{ml}$ of air at a time, then remove the sheath.

Minimal individual haemostasis (MIH)

- TR-Band inflation with $20 \mathrm{ml}$ of air at a time, then remove the sheath

- Slow deflation till the slightest sign of bleeding at the site of access

- $2 \mathrm{ml}$ of air are inflated and the total amount of air used is noted

- The same technique of slow deflation is repeated after an hour, then at an interval of 30 minutes

- TR-Band is removed as soon as there's no sign of local bleeding

- Total haemostasis time is noted

\section{Feasibility assessment}

Permeability was assessed using plethysmography and pulse oximetry, while ipsilateral ulnar artery was occluded (Barbeau inversely). Radial artery permeability has been documented if present; Otherwise, the TR band was slowly deflated until the plethysmographic signal returned to restore radial permeability. The compression flask was then further deflated until bleeding occurred or a minimum flask volume of 7 $\mathrm{ml}$ was reached. The compression flask was then refluxed $2 \mathrm{ml}$ above the bleeding point or left with a minimum of air. The TR Band remained in place for an hour and was then checked every 30 minutes until it was removed. Although bleeding was assessed, the mean time required was also calculated together with the relevant clinical data.

\section{Statistical analysis}

Data management and analysis were performed with PSPP 1.0.1 MacOS. Continuous variables are presented as mean $\pm \mathrm{SD}$ and mean are compared with the Student's t test. The categorical variables are given as proportions and compared with chi-square or Fisher. All statistical tests are two-sided and the statistical significance is defined as a $\mathrm{p}$ value $<0.05$.

\section{RESULTS}

A total of 101 patients were enrolled in the feasibility study. Data were reviewed in 101 patients. Risk factors for atherosclerotic disease, including hypertension, dyslipidemia, diabetes and smoking were present in $66 \%, 58 \%, 38 \%$ and $31 \%$, respectively. A degree of renal impairment greater than or equal to 2 was present in $27 \%$ of patients (table no. 1 ).

Table no 1. Patient's characteristics and relevant history

\begin{tabular}{|l|l|l|l|l|l|}
\hline Var & N & Mean & Std Dev & Min & Max \\
\hline Age & 101 & 63.47 & 9.38 & 45 & 82 \\
Sex & 101 & 1.38 & 0.49 & 1 & 2 \\
DM & 101 & 0.38 & 0.49 & 0 & 1 \\
\hline HTN & 101 & 0.66 & 0.47 & 0 & 1 \\
\hline Dislip & 101 & 0.58 & 0.5 & 0 & 1 \\
\hline CKD & 101 & 0.27 & 0.44 & 0 & 1 \\
Smok & 101 & 0.31 & 0.46 & 0 & 1 \\
\hline
\end{tabular}

All patients had $6 \mathrm{Fr}$ trans-radial sheaths used for classic arterial access in 50 patients and distal access in 51 patients. Percutaneous coronary intervention (PCI) was performed in $37 \%$ of patients (table no. 2 ).

Table no. 2. Incidence of PCI and medium number of stents implanted

\begin{tabular}{|c|c|c|c|}
\hline Value & Freq & Valid Percent & Cum Percent \\
\hline $\mathbf{0}$ & 64 & 63.37 & 63.37 \\
\hline $\mathbf{1}$ & 22 & 21.78 & 85.15 \\
\hline $\mathbf{2}$ & 12 & 11.88 & 97.03 \\
\hline $\mathbf{3}$ & 3 & 2.97 & 100 \\
\hline Total & 101 & 100 & \\
\hline
\end{tabular}

No bleeding complications were found and all patients were able to obtain hemostasis by this method at the initial assessment and deflation of TR Band. The local hematoma occurred in 5 patients, subsequently remitted by local cold compresses and none of them suffered radial artery occlusion or impaired forearm function.

Radial artery occlusion evaluated by 24-hour postprocedurally by ultrasonography was found in a number of 4 patients (all from the subgroup of the classical approach), with an average of $3.96 \%$, which is consistent with the minimum limit of values established by the previous studies.(3-6) The mean time required for hemostasis was $111.39 \pm 39.88$ minutes (table no. 3). In subgroup analysis, correlations were noted between both the hemostasis time and the radial artery occlusion rate and the distal transradial approach $(83.53 \pm 25.68$ minutes and $0 \%$ ). The mean length of hospital stay was $1.98 \pm 1.14$ days

Table no. 3. Mean hemostasis

\begin{tabular}{|c|c|c|c|}
\hline Value & Freq & Valid Percent & Cum Percent \\
\hline $\mathbf{6 0}$ & 23 & 22.77 & 22.77 \\
\hline $\mathbf{9 0}$ & 24 & 23.76 & 48.53 \\
\hline $\mathbf{1 2 0}$ & 25 & 24.75 & 71.29 \\
\hline $\mathbf{1 5 0}$ & 19 & 18.81 & 90.1 \\
\hline $\mathbf{1 8 0}$ & 8 & 7.92 & 98.02 \\
\hline $\mathbf{2 1 0}$ & 2 & 1.98 & 100 \\
\hline Total & 101 & 100 & \\
\hline
\end{tabular}

Five patients $(4.95 \%)$ had local hematoma after the procedure, probably due to multiple punctures. After the dressing under pressure, the hematoma improved, without ultrasonographic signs of radial artery occlusion and the activity of the hands was not affected (table no. 4).

Table no. 4. Incidence of local hematoma

\begin{tabular}{|c|c|c|c|c|}
\hline Label & Value & Freq & Valid percent & Cum Percent \\
\hline Yes & 1 & 96 & 4.95 & 4.95 \\
\hline No & 0 & 5 & 95.05 & 100 \\
\hline & Total & 101 & 100 & \\
\hline
\end{tabular}

\section{DISCUSSIONS}

This technique was associated with a minimal radial artery occlusion rate, even with the use of $6 \mathrm{Fr}$ trans-radial sheaths (even for diagnostic procedures) which are associated with higher rate of peri-procedural complications compared to smaller sizes. $(7,8)$ The feasibility of the technique was evaluated both in coronary angiography and in coronary interventions, both in the classical radial approach and in the distal one. The initial formation of the platelet plug (9) as physiological 


\section{CLINICAL ASPECTS}

haemostasis occurs may explain the shorter time of haemostasis achieved by this method.

This study did not analyse the compression pressure of the TR-band as it was not calculated initially and was very challenging to measure. Rapid deflation of the TR band reduces compression and allows patent hsemostasis, as can be confirmed by the return of the plethysmographic signal during the reverse Barbeau evaluation. While prolonged compression of the TRband is considered a direct factor for RAO permeability, we should remind that gradual deflation techniques generally require frequent training and added work for the medical team.

\section{Study limitations}

The statistical power of the study remains limited, being retrospectively analysed and with a relatively small number of patients. Radial artery occlusion was assessed only at 24 hours; previous studies have shown that a significant proportion (36-58\%) of patients will have a spontaneous resolution of radial occlusion at 30 days $* * * 10-12.15$. The technique was evaluated in a single centre with two operators, which raises the possible influence of personal factors.

\section{CONCLUSIONS}

We conclude that MIH technique as described and used is feasible after trans-radial approach, in both categories of access, conventional or distal. The rate of peri-procedural RAO was around $4 \%$ and significantly correlated to the type of approach used, with more positive results on the distal transradial approach. In addition, prospective randomised studies should be performed to confirm these findings and compare this method with other relevant techniques for radial haemostasis

\section{Impact on daily practice}

There are several protocols for deflation and elimination of the TR band that are often difficult to implement at the physician or healthcare level without standardisation and are not individualised on a patient-by-patient basis. We propose a simplification of TR Band management after performing the interventions through the classical or distal trans-radial approach. This deflation technique is safe and associated with a minimal rate of radial artery occlusion and can facilitate patient comfort and simplify the process for cardiac catheterisation and the healthcare team.

\section{REFERENCES}

1. Mehta SR, Jolly SS, Cairns J, et al. Effects of radial versus femoral artery access in patients with acute coronary syndromes with or without ST-segment elevation. J Am Coll Cardiol. 2012;60:2490-9.

2. Avdikos G, Karatasakis A, Tsoumeleas A, et al. Radial artery occlusion after transradial coronary catheterization. Cardiovasc Diagn Ther. 2017;7:305-16.

3. Pancholy SB, Patel TM. Effect of duration of hemostatic compression on radial artery occlusion after transradial access. Catheter Cardiovasc Interv. 2012;79:78-81.

4. Pancholy S, Coppola J, Patel TM, Roke-Thomas M Prevention of radial artery occlusion: patent hemostasis evaluation trial: a randomized comparison of traditional versus patency documented hemostasis after transradial catheterization. Catheter Cardiovasc Interv. 2008;72:33540.

5. Pancholy SB, Bertrans OF, Patel TM. Comparison of a priori versus provisional heparin therapy on radial artery occlusion after transradial coronary angiography and patent hemostasis (from the PHARAOH Study). Am J Cardiol. 2012;110:173-6.

6. Soydan E, Akin M. Coronary angiography using the left distal radial approach - An alternative site to conventional radial coronary angiography. Anatol J Cardiol; 2018.
7. Ahmad Edris, et al. Facilitated patent haemostasis after transradial catheterisation to reduce radial artery occlusion EuroIntervention. 2015;11:765-771.

8. Burzotta F, Trani C, Mazzari MA, et al. Vascular complications and access crossover in 10,676 transradial percutaneous coronary procedures. Am Heart J. 2012;163:230-8

9. Mehta SR, Jolly SS, Cairns J, et al. Effects of radial versus femoral artery access in patients with acute coronary syndromes with or without ST-segment elevation. J Am Coll Cardiol. 2012;60:2490-9.

10. Mattea V, Salomon C, Menck N, et al. Low rate of access site complications after transradial coronary catheterization: a prospective ultrasound study. Int J Cardiol Heart Vasc. 2017;14:46-52. 